\title{
- Dentists - An Extended Arm during COVID-19
}

\section{Dr. Little Mahendra}

IJCRR

Section: Healthcare

Sci. Journal Impact

Factor: $6.1(2018)$

ICV: 90.90 (2018)
Dean, Maktoum Bin Hamdan Dental University College (MBHDUC), Dubai.

For the past few months, the hospitals have turned into a war zone. Stress on healthcare workers is the new norm due to the unprecedented challenges across the globe as governments trying to slow the spread of the virus. The situation is still shaky and under investigation, as we learn new mischievous coronavirus behavior every day. The healthcare workers are fighting to cope with crowds of sick and nervous patients, the medical stores going out of personal protective equipment (PPE). On top of that, they are at potential risk and saving their own families to a frightening disease. Whenever our societies are threatened with situations like this, there has been always a drastic pressure and workload on the health professionals. There is a shortage of doctors, hygienists, nurses, and even non-health occupations such as administrators, cleaners, and social workers that necessitate deploying more healthcare workers to join the medical force to fight the pandemic. Having the skilled dental team as part of this strategy would increase the availability to combat the shortage.

The dental team would be a valuable addition and an excellent asset for the hospitals to the vast medical core under pressure. Dentists are effective communicators. They are also familiar with the infection control procedures, general anatomy, and work wearing PPE and are conscious of cross-infection to ensure a guarded environment for patients and other staff. Dentists have wide knowledge of basic human systems and clinical sciences. They have knowledge of not only teeth and gums but also the muscles of the head, neck and jaw, the tongue, salivary glands, the nervous system, and other areas. Besides, dentists can spot early warning signs in the mouth that may indicate systemic diseases. Dentists can recognize situations that need referring the patients for care by other specialists or physicians. They also assist in recording the medical history and symptoms and general medical wards, emergency departments, delivery of medications and therapeutic agents, and Intensive Care Unit. By having significant knowledge of adhering to prescribed treatment pathways, they provide a level ahead of care in emergencies like Pandemics, which could benefit the mainstream medical workers and ease the burden on the overall health care system.

Rather than being isolated and separated from the mainstream healthcare system, the present global crisis has made apparent that dental personnel can be integrated into the more comprehensive system. The challenge ahead is to set forth dental personnel's clinical roles in a more integrated health care model.

Key Words: COVID-19, Health care system, Dental personnel, PPE 